\title{
SGAIM: die Gründung einer neuen Fachgesellschaft
}

\author{
Jean-Michel Gaspoza, François Héritier ${ }^{\mathrm{b}}$ \\ a Prof. Dr. med., Präsident des Direktoriums der SGIM; ' ${ }^{\text {D }}$ Dr. med., Präsident der SGAM
}

Am 17. Dezember 2015 um 14 Uhr finden im Yehudi Menuhin Forum, Helvetiaplatz 6 in Bern, ausserordentliche Generalversammlungen statt, bei denen unsere beiden Gesellschaften bezüglich ihrer Auflösung Stellung nehmen werden, gefolgt von der Gründungsversammlung einer einzigen medizinischen Fachgesellschaft, der Schweizerischen Gesellschaft für Allgemeine Innere Medizin (SGAIM).

Société Suisse de Médecine Interne Générale (SSMIG), Schweizerische Gesellschaft für Allgemeine Innere Medizin (SGAIM), Società Svizzera di Medicina Interna Generale (SSMIG), Swiss Society of General Internal Medicine (SSGIM): Dies sind die Namen der Gesellschaft, zu der sich sämtliche Träger eines Facharzttitels der Allgemeinen Inneren Medizin ab dem Jahr 2016 zusammenschliessen.

Die SGAIM, die in ihren Gremien grossen Wert auf eine ausgewogene Vertretung von Ärzten aus dem ambulanten und stationären Bereich legt, übernimmt gemäss ihrer Satzung die folgenden Aufgaben:

- Erhalt und Ausweitung weitreichender Kompetenzen der Allgemeinen Inneren Medizin;

- Erhalt und Ausbau nationaler Leistungsaufträge an Weiterbildungsstätten zur Förderung einer umfassenden und qualifizierten Allgemeinen Inneren Medizin;

- Förderung von geeigneten und wirksamen Massnahmen, die den Bedürfnissen und der jeweiligen Situation der Patienten entsprechen;

- Erlass von Anwendungshinweisen bezüglich bestehender Leitlinien unter Berücksichtigung von Multimorbiditäten und Lebensqualität der Patienten;

- Entwicklung und Umsetzung von Weiter- und Fortbildung;

- Nachwuchsförderung zur Verstärkung der Allgemeinen Inneren Medizin und ihrer medizinischen Fachgesellschaft;

- Stärkung der Forschung im Bereich der ambulanten und stationären Allgemeinen Inneren Medizin

- Durchführung von Facharztprüfungen und Verwaltung der Facharzttitel;

- Organisation von Fachtagungen;
- Dialog mit der FMH, dem SIWF und anderen Ansprechpartnern (WHM, Spitäler usw.);

- Positionierung der SGAIM unter den anderen medizinischen Fachgesellschaften mit Betonung der individuellen Unterstützung multimorbider Patienten;

- Information der Mitglieder und anderer betroffener Ärzte über die Allgemeine Innere Medizin betreffende aktuelle und grundlegende Themen und Entwicklungen;

- Information der Bevölkerung, Behörden und anderer Institutionen bezüglich der Ziele und Positionen der SGAIM;

- Erbringung von Dienstleistungen zugunsten der Mitglieder;

- Anwerbung und Verwaltung der Mitglieder.

Die SGAIM wird die grösste der medizinischen Gesellschaften der FMH sein.

Die Satzung der SGAIM, deren Präambel von jungen Allgemeininternisten verfasst worden ist, wurde den Mitgliedern unserer beiden Gesellschaften mit der Einladung $\mathrm{zu}$ den genannten ausserordentlichen Generalversammlungen bereits im Oktober per Mail zugestellt. Die Statuten sind nicht nur ein juristisches Dokument sondern auch Ausdruck eines ausserordentlichen Ereignisses. Uns ist kein derartiger Präzedenzfall in der Geschichte der medizinischen Fachgesellschaften der Schweiz bekannt, die tendenziell eher dazu neigen, sich nach Teilgebieten aufzuspalten. Mit der Gründung der SGAIM soll allen Allgemeininternisten der Schweiz, ob sie im privaten oder öffentlichen Bereich, ambulant oder im Spital, in der Stadt oder auf dem Land praktizieren, die ausserordentliche und einzigartige Möglichkeit gegeben werden, einen 


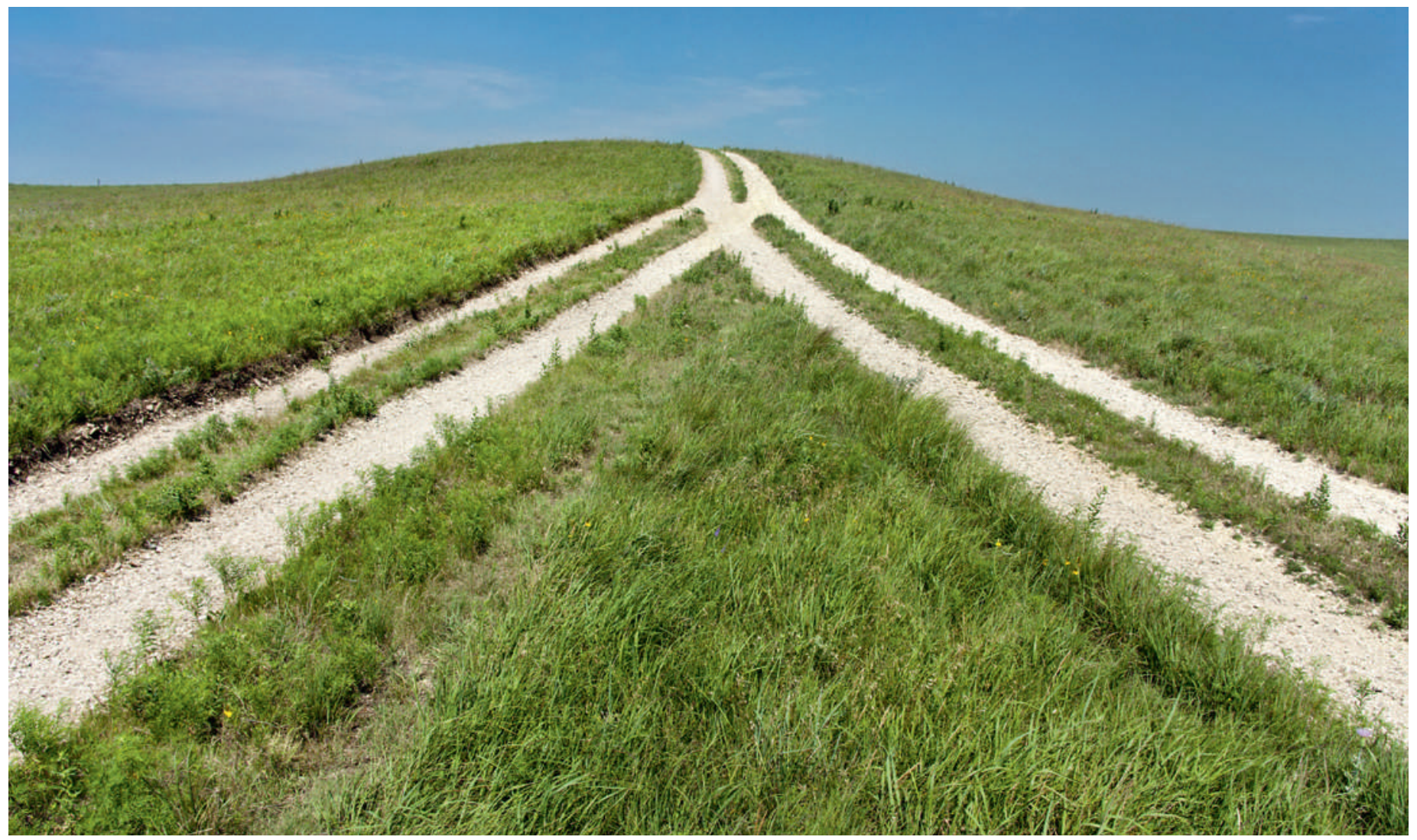

Ab dem 17. Dezember führt ein gemeinsamer Weg zum Ziel der Förderung der allgemeinen inneren Medizin.

medizinischen Fachbereich der Schweiz mit mehr als 8000 Mitgliedern zu bilden. Die SGAIM wird die grösste der medizinischen Gesellschaften der FMH sein. Dieser Zusammenschluss und seine Dynamik machen uns und den MFE, der hierdurch gestärkt wird, zu gewichtigen Akteuren bei Entscheiden, welche die Organisation und Vergütung von ärztlichen Leistungen in der Schweiz betreffen.

Die Gründung der SGAIM dient auch dem Schutz gemeinsamer Werte wie einer ganzheitlichen und patientenzentrierten Behandlung. Der Generalismus im Jahr 2015 bewahrt die Idee unserer Vorgänger, nämlich

Nehmen Sie an einem aussergewöhnlichen und historischen Ereignis in Bern teil!

nahe beim Patienten und den Familien zu sein. Es werden aber auch neue Ambitionen aufgenommen wie das Praktizieren einer leistungsfähigen, auf wissenschaftlichen Erkenntnissen basierenden Medizin, die ihr Augenmerk auf die Wirklichkeit der gegenwärtigen Patienten richtet, welche immer komplexer und polymorbider ist, und auf Menschen, die sich alleingelassen einer uneinheitlichen und zu spezialisierten Gesundheitsversorgung gegenüber sehen. Eine ganzheitliche und patientenzentrierte Behandlung beinhaltet auch die Übernahme umfassenderer Verantwortung im Hinblick auf die Gesundheitssysteme, nicht nur durch das Engagement eine kosteneffiziente Medizin zu betreiben, sondern ebenso um weitergehende Überlegungen anzustellen und an innovativen Modellen in der Gesundheitsversorgung teilzunehmen. Ist das Fachgebiet Allgemeinmedizin seiner Zeit voraus? Gewiss, denn es ist näher an den Gesundheitsproblemen unserer Mitbürger als jedes andere Fachgebiet.

Möchten Sie die Herausforderungen gemeinsam annehmen und zu unserem Fortschritt beitragen? Dann nehmen Sie am 17. Dezember 2015 an einem aussergewöhnlichen und historischen Ereignis in Bern teil.

Wir sind von der Relevanz und Notwendigkeit dieses Projekts überzeugt und freuen uns, diese spannende Herausforderung mit allen Mitgliedern unserer beiden derzeitigen Gesellschaften teilen zu können.

Bildnachweis

(c) Ricardoreitmeyer | Dreamstime.com 